\title{
GESTIÓN SOCIOAMBIENTAL SUSTENTABLE EN LA COMUNIDAD IXIL DE CHEL, CHAJUL, QUICHÉ, GUATEMALA. ESTUDIO DE CASO: HIDROELÉCTRICA COMUNITARIA
}

\author{
SUSTAINABLE SOCIO-ENVIRONMENTAL MANAGEMENT IN THE IXIL COMMUNITY, \\ CHEL, CHAJUL, QUICHÉ, GUATEMALA. CASE STUDY: HYDROELECTRIC COMMUNITY
}

GESTÃO SOCIOAMBIENTAL SUSTENTÁVEL NA COMUNIDADE IXIL DE CHEL, CHAJUL, QUICHÉ, GUATEMALA. ESTUDO DE CASO: HIDROELÉTRICA COMUNITÁRIA

\section{Candelario Elías Cruz Herrera ${ }^{1}$ \\ Estuardo Lara Ponce ${ }^{2}$ \\ Benito Ramírez Valverde ${ }^{3}$}

\begin{abstract}
Resumen
La gestión socio ambiental sirve como instrumento de diagnóstico y planificación para la resolución de los problemas medioambientales y sociales, basados en objetivos, normas, programas, estrategias, metodologías, información y educación. El objetivo del trabajo consiste en la descripción y análisis del manejo, administración, conservación y aprovechamiento del recurso hídrico para la generación de energía, por medio de la construcción de una planta hidroeléctrica en la comunidad Ixil, en Quiché, Guatemala, donde la experiencia local alcanza un bienestar social, económico y ambiental. La metodología empleada consideró un
\end{abstract}

Doi: http://dx.doi.org/10.15359/eys.23-53.6

Fecha de recepción: 09-02-2018. Fechas de reenvíos: 13-02-2018, 15-03-2018, 20-04-2018, 04-05-2018. Aceptado el 04-06-2018. Publicado el 15-06-2018.

${ }^{1}$ Egresado de la Maestría en Ciencias en Desarrollo Sustentable de los Recursos Naturales, Universidad Autónoma Intercultural de Sinaloa. Aldea Chel, Chajul él Quiché, Guatemala. Correo electrónico candelarioelias 2005@yahoo.com

2 Doctor en Estrategias para el Desarrollo Agrícola Regional, Profesor-Investigador, Universidad Autónoma Intercultural del Sinaloa, México. Correo electrónico elara@uais.edu.mx

${ }^{3}$ Doctor en Estudios Latinoamericanos, Profesor Investigador Titular, Colegio de Postgraduados, Campus Puebla, México. Correo electrónico bramirez@colpos.mx

Candelario Elías Cruz Herrera, Estuardo Lara Ponce y Benito Ramírez Valverde

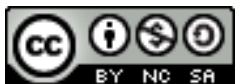

Revista Economía y Sociedad by Universidad Nacional is licensed under a CreativeCommons Reconocimiento-NoComercial- 
enfoque cualitativo descriptivo, que analizó, a través de talleres con grupos focales, el efecto de la gestión de los bienes naturales en el desarrollo generado en la comunidad. Los resultados identificaron que la Micro Central Hidroeléctrica aprovecha el potencial hídrico del arroyo Xesayí; el tipo de generación es a filo de agua, la potencia nominal de la planta es de 165 kW y la producción anual de energía es 1,23 MWh/año, la oferta de energía beneficia a 1.566 familias de 10 comunidades del Municipio de Chajul. Los instrumentos de la gestión ambiental que aseguraron el éxito de la planta comunitaria fueron la evaluación de impacto ambiental, la participación de la población y la educación; estos ejes vinculados lograron impactos favorables en lo económico, social, cultural y ambiental. Se describe el proceso de manejo, conservación y aprovechamiento de los recursos naturales, que brinda un mejor estilo de vida y de la capacidad de gobernarse a sí mismos.

Palabras clave: recursos naturales; autogestión; desarrollo sostenible; región maya.

\begin{abstract}
Socio-environmental management serves as a diagnostic and planning tool for the resolution of socio-environmental problems, based on objectives, standards, programs, strategies, methodologies, information, and education. The objective of this paper is to describe and analyze water management, administration, conservation, and use to generate energy by building a hydroelectric plant in the Ixil community, in Quiché, Guatemala, where local experience has shown social, economic, and environmental success. The methodology used a qualitative descriptive approach and analyzed, through workshops with focal groups, the effect of natural resource management in the resulting development in the community. The results identified that the hydroelectric power plant takes advantage of the water potential of the Xesayí Creek. Energy is generated from a run-of-theriver plant with a nominal power of $165 \mathrm{KW}$ and an annual energy production of 1,23 MWh/year. The energy supply benefits 1,566 families from 10 communities in the municipality of Chajul. The environmental management instruments that ensured the success of the community plant were the environmental impact assessment, population participation, and education; these linked axes achieved favorable economic, social, cultural, and environmental impacts. Management, conservation, and use of natural resources are described here, which provide a better lifestyle and the communities' ability to govern themselves.
\end{abstract}

104

Candelario Elías Cruz Herrera, Estuardo Lara Ponce y Benito Ramírez Valverde

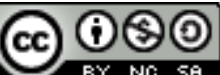

Revista Economía y Sociedad by Universidad Nacional is licensed under a CreativeCommons Reconocimiento-NoComercialCompartirlgual 4.0 Internacional License. Creado a partir de la obra en http://www.revistas.una.ac.cr/index.php/economia 
Keywords: natural resources; self-management; sustainable development; Mayan region.

\section{Resumo}

A gestão socioambiental serve como ferramenta de diagnóstico e planejamento para a resolução de problemas meio-ambientais e sociais, baseados em objetivos, normas, programas, estratégias, metodologias, informação e educação. O objetivo do trabalho consiste na descrição e análise do manejo, administração, conservação e aproveitamento dos recursos hídricos para a geração de energia, através da construção de uma usina hidrelétrica na comunidade Ixil, em Quiché, Guatemala, onde a experiência local alcança um bem-estar social, econômico e ambiental. A metodologia utilizada considerou uma abordagem qualitativa descritiva, que analisou, por meio de oficinas com grupos focais, o efeito da gestão dos recursos naturais sobre o desenvolvimento gerado na comunidade. Os resultados identificaram que a Micro Central Hidroelétrica aproveita o potencial hídrico do córrego Xesayí; o tipo de geração está no limite da água, a potência nominal da usina é de $165 \mathrm{~kW}$ e a produção anual de energia é de 1,23 MWh / ano, o fornecimento de energia beneficia 1566 famílias em 10 comunidades do município de Chajul. Os instrumentos da gestão ambiental que garantiram o sucesso da planta comunitária foram a avaliação do impacto ambiental, a participação da população e a educação; esses eixos interligados alcançaram impactos econômicos, sociais, culturais e ambientais favoráveis. Descreve-se o processo de gestão, conservação e aproveitamento dos recursos naturais, que proporciona um melhor estilo de vida e a capacidade de se autogovernar.

Palavras-chave: recursos naturais; autogoverno; desenvolvimento sustável; região maia.

\section{Introducción}

En Centroamérica, la relación de los bosques y el agua es muy estrecha (Kaimowitz, 2001). En Guatemala, país de gran diversidad cultural y biológica, el potencial energético y el nivel de aprovechamiento se basan en diversos recursos naturales como el petróleo y el consumo energético de leña, que alcanza el 57\%, y el hidroeléctrico, que solo llega al 15\%; y en el área central del país, el sector rural cuenta con $40 \%$ o menos de cobertura eléctrica, según datos de la política energética de Guatemala (2013-2027). 
Las comunidades rurales indígenas de Guatemala tienen el reto de adoptar estrategias que generen oportunidades para sus miembros y atiendan tanto el problema de injusticia social como la situación de escasez de energía eléctrica. De ahí que la gestión ambiental surge como medio social participativo, cuyo enfoque conceptual:

Inicia en la década de los setentas del siglo XX, como reacción a los graves problemas de destrucción y deterioro de los ecosistemas, su concepción significa una reorientación de parte del pensamiento ambiental y un instrumento de diagnóstico y planificación para la resolución de los problemas [...]. (Muriel, 2005, p. 1).

De acuerdo con el Instituto de Agricultura, Recursos Naturales y Ambiente y la Universidad Rafael Landívar, la gestión ambiental es "un sistema complejo de interacciones sociales, basado en objetivos, normas, programas, estrategias, metodologías, información y educación, que busca una vida en equilibrio, con relaciones armoniosas entre las personas, la comunidad, la sociedad y la madre tierra a la que pertenecemos" (2009, p. 28). Con base en esta afirmación, la planificación es el eje de la gestión ambiental que implica realizar actividades de largo plazo y desarrollar planes, programas y proyectos desde la comunidad, para generar desarrollo acorde con las características locales. La gestión ambiental, aunque es una función de la administración pública, no puede tener el éxito deseado si no considera la participación comunitaria para llevar a cabo las acciones destinadas a lograr los objetivos planteados.

Las acciones humanas colectivas aplicadas correctamente en su entorno son las que pueden establecer relaciones con la naturaleza de manera sostenida. Al respecto, Ostrom (1990), plantea que la acción colectiva se soporta en la cooperación de los individuos donde prevalece coordinación de las decisiones y acciones en torno a objetivos comunes, por lo que es una posibilidad real y contemporánea. La acción colectiva enfrenta a los participantes con costos y dilemas de sus decisiones. Se parte del supuesto de la confianza en los compromisos asumidos, al invertir en la construcción de visiones comunes sobre los bienes colectivos y lograr acuerdos en las reglas viables de cooperación; sin embargo, es una tarea social no sencilla de alcanzar, depende del contexto en que ocurra. Si los resultados no son los esperados por el colectivo, es probable que se presente el “dilema del prisionero" (Merino, 2014, p. 87).

El planteamiento de Ostrom es válido para el presente caso de estudio. La aplicación de los instrumentos de la gestión ambiental colectiva aseguró el éxito de la planta hidroeléctrica comunitaria chelense, mediante la implementación de tres componentes vinculados: a) la evaluación de impacto ambiental; b) la participación de la población; y 3) la educación y formación ambiental de los sujetos. Estos instrumentos son los elementos operativos para la protección y garantía de la preservación del medioambiente en la comunidad, con apego a un marco legal establecido (Ley de Protección y Mejoramiento del Medio Ambiente, 1986).

106

Candelario Elías Cruz Herrera, Estuardo Lara Ponce y Benito Ramírez Valverde

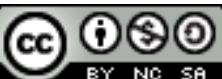

Revista Economía y Sociedad by Universidad Nacional is licensed under a CreativeCommons Reconocimiento-NoComercial- 
En Guatemala, la comunidad Ixil de Chel habita una región privilegiada, rica en recursos naturales y culturales de raíces ancestrales; sin embargo, como sucede en muchas comunidades rurales del país, necesitan de fuentes de energía para su vida cotidiana. Los pobladores resolvieron la situación con la implementación de una hidroeléctrica comunitaria. Según la Organización Latinoamericana de Energía (OLADE), la generación de energía a escala local, comparada a nivel de región o país, no es significativa, porque "es un proyecto aislado que no está interconectado con el sistema nacional de electrificación y es totalmente comunitario [...] de enorme valor socioeconómico y ambiental, que indujo un proceso de cambio social local, como en las comunidades aledañas de extrema pobreza" (OLADE, 2012, p. 46).

El objetivo del estudio fue el análisis de la información, manejo, administración, conservación y aprovechamiento del agua para la generación de energía, por medio de la construcción de una Micro Central Hidroeléctrica ( $\mathrm{MCH}$ ) en la comunidad Ixil, de Chel, Chajul, Quiché, Guatemala. Se identificaron los componentes que facilitaron la gestión socioambiental del recurso hídrico, teniendo como antecedente las condiciones precarias iniciales y los efectos posconflicto armado en la población. Los elementos exitosos detectados son de utilidad para los gestores del sector público y privado y un medio de información de los conocimientos sobre este tipo de acciones comunitarias.

\section{Orígenes del pueblo maya Ixil}

El pueblo Ixil desciende de los antiguos mayas que habitaron la mayor parte del territorio de Guatemala. Se cree que los antiguos Ixiles eran provenientes de tierras bajas del mirador; Peten (900 a.C. - 200 d.C.). En el 200 d.C. cae el mirador y surge Tikal como ciudad Estado; los ixiles antiguos se asentaron en las faldas del cerro llóm 500 años antes de Cristo. En este lugar fueron afectados por una epidemia que los obligó a emigrar en búsqueda de nuevos horizontes y tomaron tres direcciones. Algunos emigraron a la parte central Xo'l laq jul (Chajul), otro grupo emigró a la zona cálida suroriental Tz'a'la Vitz, (Cotzal) y el último se dirigió a las orillas de la laguna Naab'a' (Nebaj) al sur occidente. (Academia de Lenguas Mayas de Guatemala, 2008, p. 17)

La cultura ixil de la aldea Chel pertenece a las comunidades mayas del altiplano de Guatemala, forman parte de la inmensa área cultural mesoamericana que mantiene una identidad compartida. Geográficamente el área se extiende de los límites del norte de Sinaloa, Nayarit, Zacatecas, San Luis Potosí y la frontera de Veracruz con Tamaulipas al norte de México, prolongándose hasta los territorios de Guatemala, Belice, El Salvador y Honduras en Centroamérica (Akkeren, 2005, p. 07). 


\section{Descripción y ubicación geográfica del área de estudio: aldea Chel}

Guatemala, del náhuatl Quauhtlemallan, "lugar de muchos árboles", cuenta con una superficie territorial de 108,889 km², distribuidos política y administrativamente en 22 departamentos. La comunidad Ixil de Chel forma parte de las 63 comunidades que integran el Municipio de Chajul, Departamento de Quiché, en la región Ixil, que incluye a Nebaj, Cotzal y Chajul. Geográficamente se localiza en las coordenadas $15^{\circ} 38^{\prime} 25^{\prime \prime}$ latitud norte y $91^{\circ} 04^{\prime} 08^{\prime \prime}$ longitud oeste, en las estribaciones de las montañas de la Sierra de Chamá, que forma parte del área de usos múltiples de la Reserva Protegida Visís-Cabá, junto a la rivera del río que lleva el mismo nombre, a una altura de 900 metros sobre el nivel del mar (Gall, 1976, p. 636) (Figura 1 ).

La comunidad Chel, colinda al Norte con el municipio de Ixcan, al Oeste con la aldea de Ilóm, al Este con el municipio de Uspantán, al Noroeste con la aldea de Amajchel y al sur con la cabecera municipal de San Gaspar Chajul. Internamente el polígono territorial se divide en cinco zonas, dista a $39 \mathrm{~km}$ de la cabecera municipal y $315 \mathrm{~km}$ de la ciudad capital. El acceso es por la ruta Guatemala-Santa Cruz-Sacapulas, Nebaj tramo asfaltado de 280 km; hasta llegar al Municipio de Chajul, luego se viajan 39 $\mathrm{km}$ en carretera de terracería en mal estado. El tiempo de viaje ininterrumpido hasta la ciudad capital dura alrededor de 10 horas. (Fundación Solar, 2010, pp. 8-9)

\section{Aspectos ambientales regionales}

El clima es templado, lluvioso de junio a diciembre. La precipitación pluvial oscila de 2.000 a $3,000 \mathrm{~mm}$ y es abundante de mayo a diciembre. Las tierras frías se encuentran en altitudes de 1.500 a 3.000 msnm, con temperaturas entre 10 y $17^{\circ} \mathrm{C}$. Las llamadas tierras "calientes" por los ixiles presentan altitudes de 800 a 1.500 msnm (Durocher, 2002).

La principal cuenca hidrográfica que drena la región es el río Xacbal, cuya vertiente pertenece a la cuenca del Golfo de México. Convergen además nueve ríos en el área, hacia el norte río Cabá, al noroccidente río Chel y Xacbal, al suroccidente río Pumila, al centro río San Vicente y al oriente río Putul y río Cancab (Rey, Conde, Godoy, Secaira y Schuster, 1996). Los suelos de la región son arcillosos, localizados en relieves ondulados, inclinados y quebrados, susceptibles a la erosión, con pendientes del 4 al 32\%.

La comunidad de Chel colinda al este con la biósfera Visís-Cabá, que cubre una extensión de 45.000 hectáreas. En términos ecológicos, es la tercera reserva biosfera más grande del país, con presencia de bosque tropical lluvioso en región húmeda, con nueve ríos e innumerables riachuelos y nacimientos de agua, razón por la que se clasifica como selva tropical lluviosa (Holdridge, 1982). De acuerdo con el Centro de Acción Legal Ambiental y Social de Guatemala (CALAS), la reserva forma parte de la Sierra de Chamá, situada en la región montañosa entre 108

Candelario Elías Cruz Herrera, Estuardo Lara Ponce y Benito Ramírez Valverde

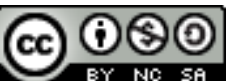

Revista Economía y Sociedad by Universidad Nacional is licensed under a CreativeCommons Reconocimiento-NoComercial- 
Tijuil y Cabá en el municipio de Chajul; posee elevaciones de 700 a 3.300 msnm. En su alrededor se encuentran las comunidades Chel, Xesayí, Juá, Bichox, Visiquichum, Juil, Vitzích, Cabá, Pal, Xaxboq, Chexá y Santa Rosa (CALAS, 2004; Durocher, 2002). Esta área de biodiversidad alberga una enorme cantidad de especies endémicas de fauna (Área de Derechos Colectivos Ambientales de Pueblos Indígenas, 2006).

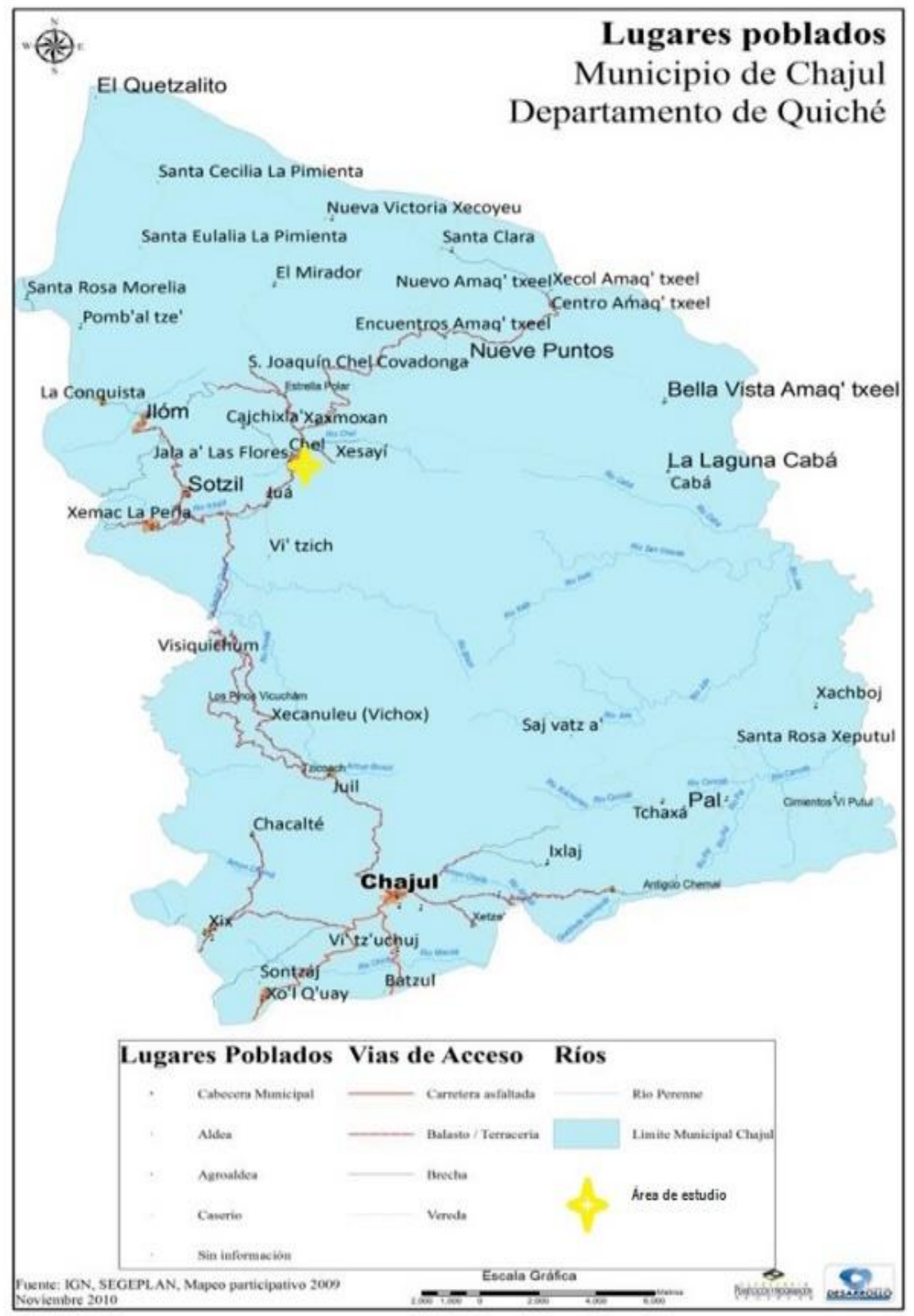

Figura 1. Localización geográfica de la Comunidad de Chel, municipio de Chajul, Quiché. Guatemala. Fuente: Consejo Municipal de Desarrollo (2010).

Candelario Elías Cruz Herrera, Estuardo Lara Ponce y Benito Ramírez Valverde

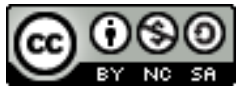

Revista Economía y Sociedad by Universidad Nacional is licensed under a CreativeCommons Reconocimiento-NoComercial- 


\section{Población y situación sociocultural}

La población en el 2017, según el Censo del Puesto de Salud de Chel, contabilizaba 2,761 habitantes, de los cuales 1.433 eran hombres (51,90\%), y 1.328 mujeres (48,10\%). La edad productiva de la población de Chel, en los rangos de edades de 10 a 15 y de 15 a 20 años, significa un gran potencial de desarrollo del capital humano.

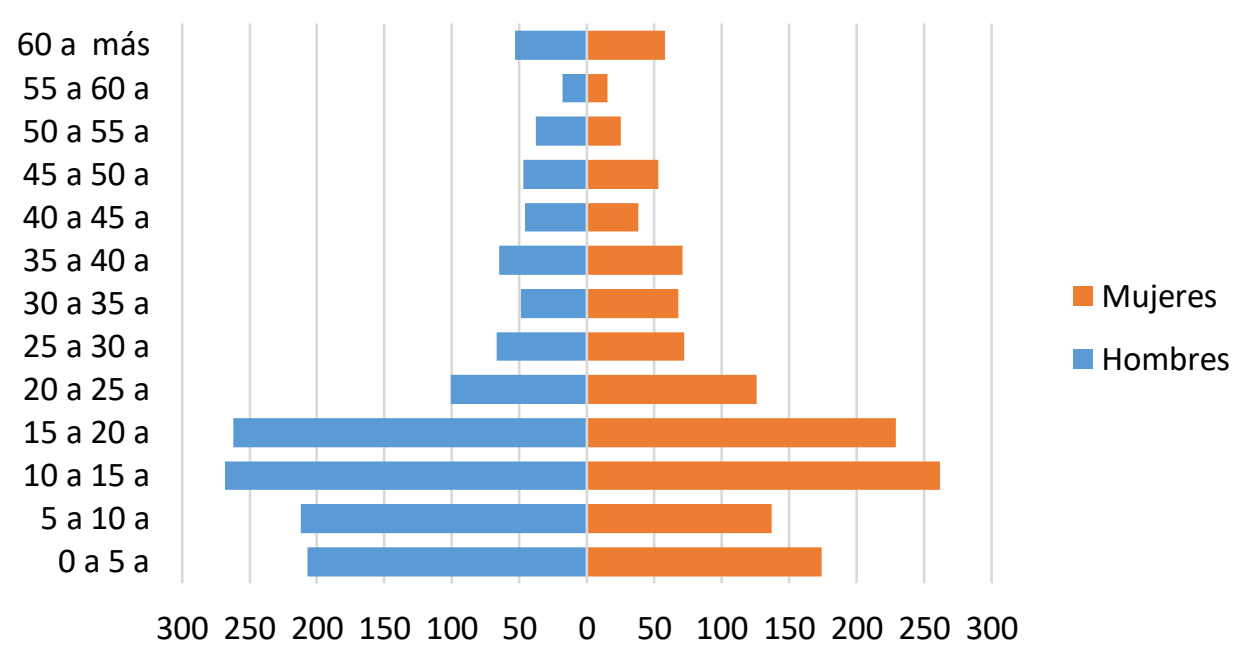

Figura 2. Pirámide poblacional aldea Chel, 2017. Fuente: elaboración propia con datos del Ministerio de Salud Pública y Asistencia Social (MSPAS, 2017).

\section{Educación y salud}

La educación formal inició en 1988, con una escuela bilingüe, patrocinada por voluntarios de los Estados Unidos, a través de la iglesia Jesús Sana y Salva. Las clases se impartían en una casa de madera en idioma ixil. Al ser una comunidad rural marginada, la educación no era accesible para todos, las limitaciones económicas obligaban a los infantes a trabajar a temprana edad, lo que arrojaba índices reprobatorios y deserción escolar. Actualmente, la comunidad de Chel cuenta con los sistemas educativos básicos de preprimaria, primaria, básico y educación media.

En materia de salud, la cobertura actual permite a la comunidad su acceso a esta. El servicio lo brinda el Ministerio de Salud Pública y Asistencia Social del Municipio de Chajul. Se atiende a una población de 350 a 500 habitantes al año. La Dirección de Salud Ixil reportó en el 2017 que las causas de morbilidad general e infantil fueron las infecciones respiratorias, enfermedades estomacales e infección de la piel. 


\section{Religión, costumbres y tradiciones}

Los habitantes de Chel, en la época colonial y antes del conflicto armado, eran predominantemente católicos (De León, 2006). En la actualidad, proliferan las iglesias evangélicas (Dios Viviente, Jesús Sana y Salva, Vida Cristiana, Evangelio Completo, entre otras). El municipio de Chajul conserva su riqueza cultural y natural; y en la comunidad de Chel prevalecen tradiciones a pesar de las adversidades del conflicto de los ochentas. Continúan modos de vida y de pensar propios de la cultura ixil, como las prácticas y celebraciones dedicadas al bienestar, la vivienda, la siembra, la cosecha, la Madre Naturaleza y la Tierra, la memoria de sus difuntos y la celebración de la culminación del año solar (Akkeren, 2005).

\section{Economía y actividades productivas}

El sustento económico de los ixiles es la agricultura de temporal. Los cultivos tradicionales son maíz y frijol, mientras que las plantaciones de café y cardamomo (Elettaria cardamomum), generan los principales ingresos de la población. El café que se cosecha y procesa de manera orgánica se exporta a Estados Unidos y Europa a través de la agrupación de productores "Asociación Chajulense", obteniendo así un precio justo. Los pobladores que no pertenecen a dicha asociación lo venden a intermediarios de la región; situación similar sucede con el cardamomo. Las familias dentro de las parcelas de café producen plátano, frutales y hortalizas; así como ganadería familiar con cabezas de cerdos y, escasamente, ganado vacuno.

\section{Hidroeléctrica en beneficio de Chel}

De acuerdo con Molina (2012), los programas de electrificación rural oficiales no llegaron a beneficiar poblaciones en condiciones de extrema pobreza. Fueron instituciones externas, como Fundación Solar, las que impulsaron proyectos de mediano tamaño para usos productivos de energía eléctrica con medios renovables y trabajaron con dos poblaciones aisladas, una de ellas la aldea Chel.

El proyecto local es considerado como una hidroeléctrica sin conflictividad que genera bienestar a la población aledaña (Molina, 2012). Sin embargo para comprobar lo anterior surgió el interés por profundizar en la experiencia comunitaria exitosa y responder a las siguientes interrogantes ¿cómo los habitantes han logrado su desarrollo comunitario a partir de su historia reciente?, ¿de qué manera la gestión ambiental ha impulsado la mejora en la calidad de vida de los pobladores? y ¿cuáles han sido los impactos y beneficios logrados por la generación de energía de la $\mathrm{MCH}$ en Chel y otras comunidades? 


\section{Metodología}

Se realizó una investigación descriptiva de enfoque cualitativo, considerando a la comunidad de Chel como estudio de caso. El trabajo documental inició con la revisión de publicaciones del ámbito local y diversas consultas a fuentes secundarias que proporcionaron información para contextualizar el marco de referencia del área de estudio. La etapa de campo abarcó el año 2017, cuando se realizaron alrededor de 15 entrevistas a informantes clave de las instituciones involucradas en el proyecto hidroeléctrico. Se inició a partir de una reunión informativa con miembros del Consejo Comunitario de Desarrollo (COCODE), organización legalmente reconocida por la legislación guatemalteca, que promueve el desarrollo comunitario y el involucramiento de la población. Posteriormente, se realizaron dos talleres participativos, con la inclusión de representantes de cada agrupación existente en la comunidad, denominados grupos focales. Esta técnica de trabajo grupal propuesta por Hamui-Sutton y Valera-Ruiz (2013), sirvió para recabar información primaria confiable mediante la aplicación de un instrumento guía de trabajo previamente diseñado. El listado de los actores sociales que participaron en los talleres y el tipo de agrupación que representan se muestran en las Tablas $\underline{1}$ y $\underline{2}$.

La observación participante (Kawulich, 2005) fue otra herramienta empleada durante la investigación; de ella se obtuvieron elementos de juicio para analizar las dinámicas productivas, sociales, económicas, culturales, ambientales de la comunidad, incluyendo los conocimientos y experiencias de vida de los habitantes. El primer autor del estudio es oriundo de la región y de identidad ixil, razón por la que se facilitó la etapa de trabajo de campo y el acceso directo a Chel. Desde hace varios años se han estrechado vínculos con las autoridades comunitarias, líderes, representantes de comités, especialistas espirituales, entre otros.

\section{Tabla 1}

Lista de participantes Grupo Focal No. 1. Aldea Chel, 2017.

\begin{tabular}{clcl}
\hline No. & \multicolumn{1}{c}{ Agrupación que representa } & Sexo & \multicolumn{2}{c}{ Cargo } \\
\hline 1 & Comité de Agua & $\mathrm{M}$ & Presidente \\
2 & Puesto de Salud & $\mathrm{F}$ & Encargada \\
3 & Comité de Agua & $\mathrm{M}$ & Tesorero \\
4 & Consejo Comunitario de Desarrollo (COCODE) & $\mathrm{M}$ & Presidente \\
5 & Consejo Comunitario de Desarrollo (COCODE) & $\mathrm{M}$ & Secretario \\
6 & Alcaldía Comunitaria & $\mathrm{M}$ & Alcalde Comunitario \\
7 & Alcaldía Comunitaria & $\mathrm{M}$ & Regidor \\
8 & Comité de Salud & $\mathrm{M}$ & Presidente \\
9 & Comité de Salud & $\mathrm{M}$ & Secretario \\
10 & Alcaldía Indígena & $\mathrm{M}$ & Alcalde \\
\hline
\end{tabular}

Fuente: elaboración propia.

112

Candelario Elías Cruz Herrera, Estuardo Lara Ponce y Benito Ramírez Valverde

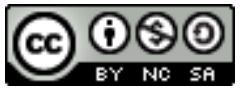

Revista Economía y Sociedad by Universidad Nacional is licensed under a CreativeCommons Reconocimiento-NoComercial- 
Tabla 2.

Lista de participantes "Grupo Focal" No. 2. Aldea Chel, 2017.

\begin{tabular}{clcc}
\hline No. & \multicolumn{1}{c}{ Agrupación que representa } & Sexo & Cargo \\
\hline 1 & Alcaldía Indígena & $\mathrm{M}$ & Vice-Alcalde \\
2 & Asociación Hidroeléctrica Chelense & $\mathrm{M}$ & Presidente \\
3 & Asociación Hidroeléctrica Chelense & $\mathrm{M}$ & Gerente \\
4 & Centro de Formación C\&N & $\mathrm{F}$ & Directora \\
5 & Iglesía Evangelio Completo & $\mathrm{M}$ & Pastor \\
6 & Cooperativa Flor de Café & $\mathrm{M}$ & Presidente \\
7 & Asociación Bilingüe Intercultural Maya Ixil Kajb’al No'j & $\mathrm{M}$ & Presidente \\
8 & Escuela Oficial Rural Mixta Chel. & $\mathrm{M}$ & Director \\
\hline
\end{tabular}

Fuente: elaboración propia.

\section{Estructuración de las actividades de los talleres}

Como producto de los talleres realizados con cada grupo focal, se trabajó en el análisis de la información, como son las variables sociales, económicas y ambientales. La información obtenida en el contexto grupal de los talleres, se utilizó para explicar las características de la planta hidroeléctrica comunitaria y actividades como el empleo de recursos agroforestales, así como las acciones que los comunitarios mantienen con la naturaleza con una visión de sostenibilidad.

\section{RESULTADOS Y DISCUSIÓN}

\section{Desarrollo comunitario basado en la gestión ambiental}

La Oficina de Derechos Humanos del Arzobispado de Guatemala (ODHAG) refiere que los aspectos históricos locales iniciaron en 1996, tras la firma de los acuerdos de paz en Guatemala. Los ixiles de la Aldea Chel, tuvieron que usar las armas para defender su vida y territorio durante el conflicto armado en la década de 1980 (ODHAG, 2000). De esta cruda experiencia, los habitantes comprendieron que comenzaba el tiempo para trabajar por el bien de la comunidad, formaron un Comité Pro mejoramiento para construir un camino de paz y desarrollo. En 1997, los pobladores contaban con el Comité como única vía de reivindicación, el fin era gestionar proyectos como la introducción de agua potable, drenaje y energía eléctrica. Gracias al apoyo de la municipalidad de Chajul y a las gestiones con la Asociación Pro agua del Pueblo, se logró la introducción de agua potable.

En Chel los afluentes de agua son diversos y presentan un caudal suficiente por la buena cobertura forestal que proviene de la reserva biósfera Visís-Cabá, un espacio natural con una

Candelario Elías Cruz Herrera, Estuardo Lara Ponce y Benito Ramírez Valverde

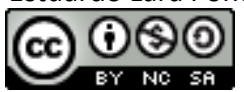

Revista Economía y Sociedad by Universidad Nacional is licensed under a CreativeCommons Reconocimiento-NoComercial- 
extensión de 45.000 hectáreas, misma que fue declarada área protegida en julio de 1997 (decreto No. 40-97 del Congreso de la República). En esta reserva se localizan bosques de uso comunal (CALAS, 2004), que se encuentran densos en cobertura debido al manejo que los pobladores ixiles le han dado a las condiciones naturales, como la topografía accidentada de grandes pendientes, y a los tipos de suelos pedregosos (Ministerio de Cultura y Deportes de Guatemala, 2001). La vegetación pertenece al sistema montañoso de ambiente húmedo, con ecosistemas nublados, vegetación cuantiosa y variada. Las coníferas son predominantes en este bioma, con presencia de Pinus, y otras especies. Este bioma recibe humedad de los vientos alisios, se condensa gran parte de niebla y, en consecuencia, es un servicio ambiental productor de agua en la región (Rey et al., 1996). Las condiciones ecológicas son razones suficientes que dieron soporte a la construcción de la planta hidroeléctrica.

En Chel se tuvieron que asegurar medidas comunitarias para continuar con la tradición de respeto a la naturaleza. De ahí el apoyo del Comité responsable de mantener el cuidado del agua, el manejo de nacimientos y la siembra de especies de uso agroforestal, como el árbol cushin (Inga spuria) y el banano (Musa paradisiaca). Desde entonces, periódicamente, los habitantes dan limpieza a los tanques de captación del agua y existen castigos para la persona que la contamine. Al inicio de estas actividades se logró la construcción de letrinas con fondos de inversión social y se construyó una pequeña unidad de Salud.

\section{Generación de energía hidroeléctrica}

El retorno de los ixiles de Chel, que estuvieron bajo las montañas resguardando su vida, tuvo resultados positivos; el lugar donde retornaron temporalmente después del conflicto armado (Finca La Perla), contaba con una minihidroeléctrica, ello generó la inquietud dentro del Comité Pro Mejoramiento para conseguir su propia energía. En 1998, por medio del municipio de Chajul, se contactó a la ONG Fundación Solar, para la construcción de una MCH comunitaria. Las condiciones para concretar dicho proyecto no eran alentadoras, se tuvieron que enfrentar una serie de retos como una infraestructura vial inexistente o que la carretera de terracería llegaba hasta el lugar denominado Visiquichum que implicaba caminatas de cuatro horas. En la aldea no existía una estructura organizativa como persona jurídica, para que adquiriera la capacidad de construir la infraestructura, administrara de la $\mathrm{MCH}$ y encargara de la operación y mantenimiento del equipo.

De acuerdo con la Comisión para el Esclarecimiento Histórico (CEH), la situación social era problemática, los niveles de escolaridad eran bajos, la pobreza era alta, la escuela de la comunidad era de madera con piso de tierra; la infraestructura para servicios de salud estaba deficiente y en mal estado, solamente se contaba con un promotor de salud. En la memoria colectiva prevalecía la desconfianza debido a las 97 personas masacradas durante el conflicto armado (CEH, 1999, p. 63).

114

Candelario Elías Cruz Herrera, Estuardo Lara Ponce y Benito Ramírez Valverde

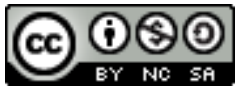

Revista Economía y Sociedad by Universidad Nacional is licensed under a CreativeCommons Reconocimiento-NoComercial- 
Entre 1998 y 2000, los vínculos del Comité Pro Mejoramiento y Fundación Solar se consolidaron y se realizaron los primeros estudios y prospección comunitaria para determinar el caudal del río Chel y del arroyo Xesayí, a efecto de estimar la capacidad de generación, calcular la demanda y la disposición de los comunitarios para desarrollar el proyecto de la $\mathrm{MCH}$. En la construcción se contó con asistencia técnica y financiera de diversas instituciones como: Fundación Solar, Instituto Humanista para la Cooperación, Agencia de los Estados Unidos para el Desarrollo, Fondo de Inversión Social, Fondo Nacional para la Paz, Green Empowerment, Instituto Nacional de Electrificación, Organización de Estados Americanos, Municipalidad de Chajul, entre otros organismos.

De las instituciones involucradas, Fundación Solar jugó un papel importante en el proceso de fortalecimiento comunitario; brindó capacitación, trabajó con líderes comunitarios temas de planificación, autogestión, administración, finanzas, organización y empresarialidad, a efecto de que comprendieran y aceptaran los cambios futuros que llevaría la instalación del servicio de energía eléctrica. Se abordaron temas de sensibilización y se resolvieron conflictos para que los comunitarios aportaran su mano de obra no calificada.

\section{El papel de la población local}

En 1999, en la aldea Chel había 350 casas, habitadas por unas 400 familias. Las secuelas del conflicto dejaron su huella en la estructura de la población, como puede apreciarse en la pirámide poblacional con datos de finales del año 2000 (Figura $\underline{3}$ ).

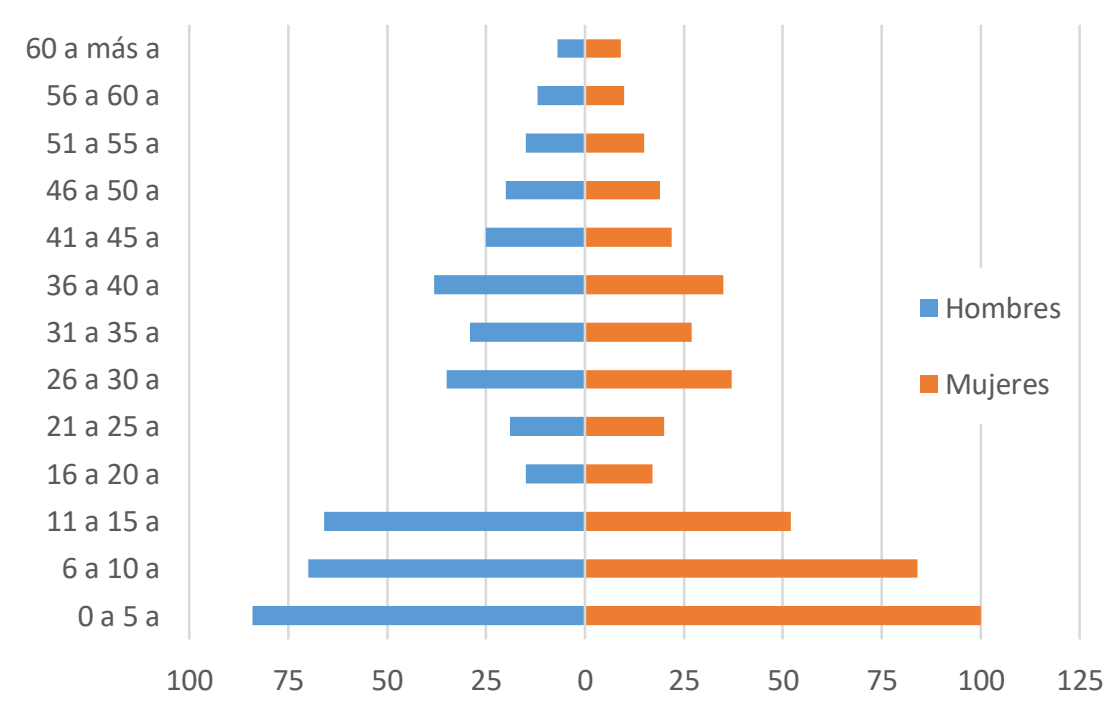

Figura 3. Pirámide poblacional de la aldea Chel, 1999. Fuente: Informe del Censo socioeconómico: Comunidades de Chel, Jala, Las Flores y Xezayí, municipio de Chajul, Departamento de Quiche (Fundación Solar, 2010).

Candelario Elías Cruz Herrera, Estuardo Lara Ponce y Benito Ramírez Valverde 
Existe una brecha poblacional en el rango de edades de 16 a 20 y de 21 a 25 años, ocasionada por la masacre en la Guerra Civil; sin embargo, a pesar de esta declinación poblacional, el deseo de superación, la forma tradicional de organización comunitaria y ayuda mutua, entendida como trabajo en equipo, no se perdieron. Estas prácticas culturales de identidad ixil, revitalizaron la fuerza de trabajo local para trabajar en la brecha de camino y la mejora del acceso peatonal a la comunidad.

En 2001 se creó la Asociación Hidroeléctrica Chelense (ASOCHEL), con el propósito de operar la $\mathrm{MCH}$, se promovió la rearticulación del tejido social y el manejo y protección de los recursos naturales, al adoptar la energía renovable a través de la participación organizada en la planificación, gestión, construcción y operación de la $\mathrm{MCH}$. El organigrama de la ASOCHEL en funciones se muestra a continuación en la Figura $\underline{4}$.

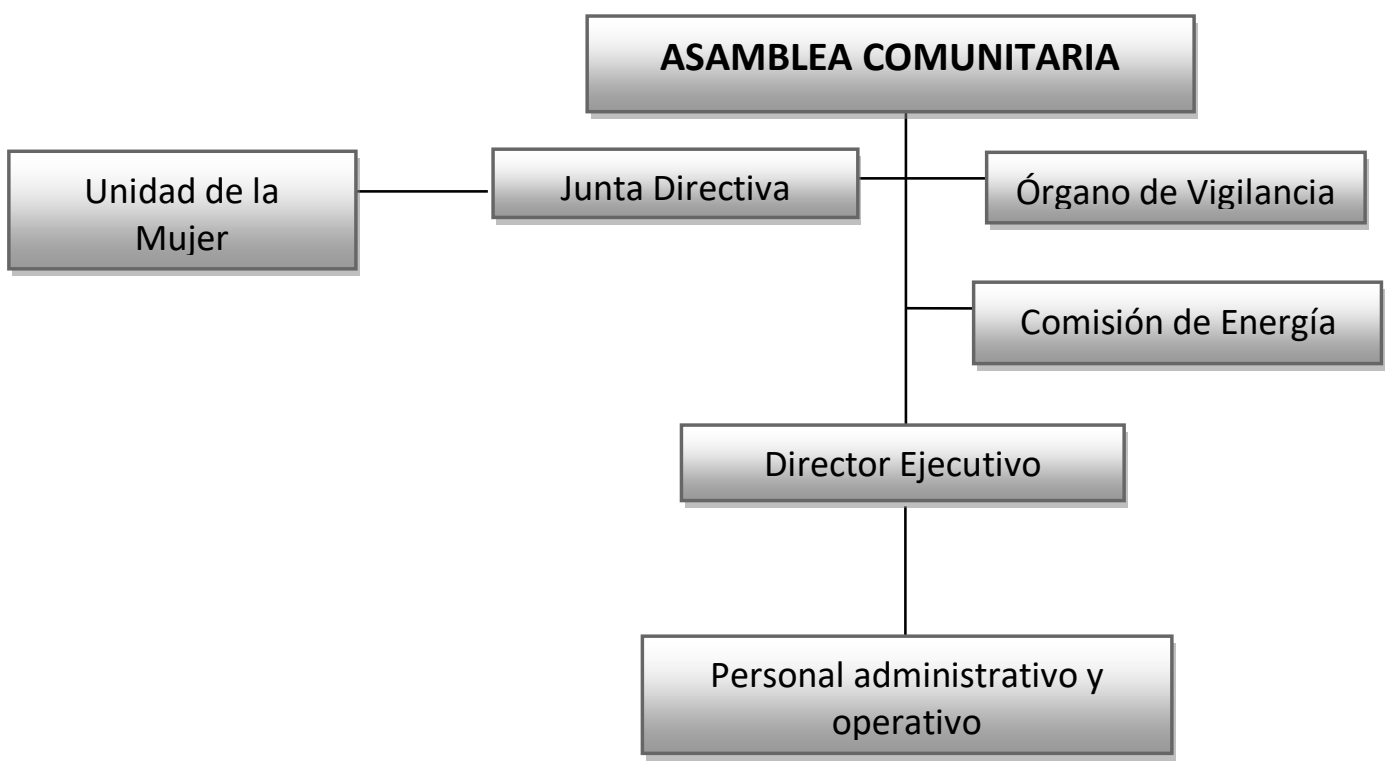

Figura 4. Organigrama funcional de la ASOCHEL. Fuente: elaboración propia con datos obtenidos en la ASOCHEL (2017).

Para impulsar la $\mathrm{MCH}$ en Chel, la Asociación requirió de la organización de la mano de obra local, mediante la contribución de 80 días de trabajo por familia en el proyecto, lo que dio derecho de conexión a la red local $(13,5 \mathrm{~km})$ y a la instalación domiciliar. Los trabajos comenzaron en 1998 y culminaron en el 2007. Inicialmente, el proyecto de la MCH ofertó el servicio energético eléctrico a 350 familias de las aldeas Chel, Jalá, Las Flores y Xesayí, del municipio de Chajul. Sin embargo, dos años más tarde la demanda y aceptación del proyecto se incrementó en la región y con el apoyo financiero del Proyecto de Desarrollo Rural (PRORURAL) 
e Hidroxacbal pasó a atender de tres a un total de 10 comunidades, con lo que a finales del año 2017 se benefició un total de 1.566 familias (Tabla $\underline{3}$ ).

Tabla 3.

Comunidades y familias atendidas por la Asociación Hidroeléctrica Chelense, 2017.

\begin{tabular}{llr}
\hline No. & Comunidades & Familias \\
\hline 1 & Chel & 378 \\
2 & Xesayí & 17 \\
3 & Jala á Las Flores & 100 \\
4 & Cajchixla & 27 \\
5 & Estrella Polar & 140 \\
6 & Cobadonga & 28 \\
7 & Jua & 137 \\
8 & Sotzil & 241 \\
9 & llóm & 422 \\
10 & Xaxmoxan & 76 \\
Total & & 1566 \\
\hline
\end{tabular}

Fuente: modificado de ASOCHEL (2017).

\section{Escenario de éxito de la MCH Chelense}

Los líderes y directivos de la ASOCHEL coinciden en que para mantener la sostenibilidad del proyecto y responder a la demanda creciente de los usuarios, los escenarios a corto plazo son: interconectarse y vender los excedentes de energía eléctrica; a mediano y largo plazo, comprar energía al Sistema Nacional Interconectado para cubrir el incremento de la demanda proyectada. Sin embargo, a partir del 2012 se ha presentado la escasez del recurso hídrico en los meses de verano (marzo y abril), lo que provoca cortes en el servicio en "horas pico" (18:00 a 20:00 horas); esto es atribuible posiblemente a los efectos del cambio climático global. Para paliar la escasez de agua en tiempo de verano, se está construyendo una cámara de carga con capacidad para almacenar $2700 \mathrm{~m}^{3}$ de agua, para ser utilizada en el tiempo de verano, con un costo de Q 385.000 .00 (equivalente a USD 50.000) con fondos propios de la ASOCHEL.

En el plan estratégico de la ASOCHEL, se establecen otras alternativas para satisfacer la demanda de energía futura, además de la interconexión, las cuales se resumen en tres acciones comunitarias: a) mejorar la eficiencia del equipo de generación y optimizar el diseño de la obra civil; b) construcción de una $\mathrm{MCH}$ en cascada para aprovechar el desfogue de la casa de máquinas, con base en un estudio de factibilidad; y c) realizar un proyecto de generación sobre el río Chel, a la altura del puente de ingreso a la comunidad, a través de alianzas públicoprivadas. 
Vale la pena mencionar que para la alternativa c), la ASOCHEL cuenta con los estudios de prefactibilidad técnica y económica del proyecto hidroeléctrico, dicho estudio identificó una alternativa viable de generación de $8,07 \mathrm{MW}$, se plantea desarrollar un proyecto bajo un modelo de co-inversión con participación privada y comunitaria; sin embargo, a la fecha se está definiendo el plan de negocios bajo un esquema de privado-comunitario para negociar con los inversionistas interesados.

La $\mathrm{MCH}$, con capacidad de generación de 165 kW, no está interconectado al Sistema Nacional Eléctrico, es totalmente comunitario y tiene un valor socioeconómico y cultural importante, porque se identifica como un proyecto de cambio sociocultural y de impacto en el nivel de vida de comunidades en situación vulnerable. La comunidad Chel, previo a la operación de la $\mathrm{MCH}$, presentaba marginación social con altos índices de analfabetismo, bajo nivel de escolaridad, alta tasa de mortalidad infantil, escasez de servicios e infraestructura de electrificación, altos niveles de conflictividad social y presión de los recursos naturales por el avance de la frontera agrícola (OLADE, 2012).

Es una ventaja que Chel disponga de la $\mathrm{MCH}$, pues es la encargada de generación, distribución y administración de la energía eléctrica, coordina la toma de decisiones, favorece la dirección acorde a sus valores culturales, alternando con formas actuales de liderazgo, y se establece los procedimientos democráticos, convirtiéndose en una organización que ha favorecido la gestión comunitaria con proyectos de beneficio colectivo.

Para la interconexión de las nuevas comunidades a la $\mathrm{MCH}$, la Hidroeléctrica Xacbal construyó una red de distribución de energía eléctrica de 27 kilómetros de 34,5 Kw y PRORURAL construyó $14,5 \mathrm{~km}$ de red de distribución de baja tensión, con un costo aproximado de 4,3 y 4,5 millones de Quetzales, beneficiando a más de mil familias de las comunidades de Xaxmoxan, Cajchixlá, Estrella Polar, Covadonga, Ilóm Sotzil y Jua. Durante el periodo 2016-2017, la Hidroeléctrica Xacbal, instalada en el Municipio de Chajul con capacidad de generación de $94 \mathrm{MW}$, ha liderado acciones encaminadas a mejorar las condiciones forestales de la región. En el año 2016 totalizó la plantación de 68.309 individuos de diversas especies nativas, entre forestales, frutales y agroforestales, logrando la reforestación de 49.98 hectáreas; para el 2017, se plantó un total de 118.236 ejemplares reforestando 92,03 hectáreas, la reforestación total fue de 142,01 hectáreas. Estas acciones comunitarias buscan la protección de la cuenca alta y media del rio Xacbal y de las microcuencas del rio Chel y Xesayí. El objetivo es contribuir a las prácticas de adaptación y mitigación al cambio climático que se percibe en las comunidades de la región.

118

Candelario Elías Cruz Herrera, Estuardo Lara Ponce y Benito Ramírez Valverde

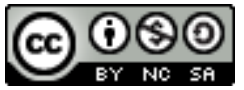

Revista Economía y Sociedad by Universidad Nacional is licensed under a CreativeCommons Reconocimiento-NoComercial- 


\section{Impactos del proyecto $\mathrm{MCH}$}

La visión del desarrollo desde la cultura y el nivel de conocimiento alcanzado por los ixiles se enmarca en la cosmovisión maya y en la práctica social. Todas las estructuras de la sociedad sean políticas, económicas, sociales, culturales y religiosas, obedecieron un mismo patrón y, en cada una de ellas, se aplicó la cooperación como ley fundamental del desarrollo humano, generando como sistema propio el comunitarismo, esencia del modelo de vida que ha generado la plataforma del desarrollo sostenible del futuro.

Con base en la práctica de los conocimientos adquiridos, se originaron impactos en las áreas económica, social, cultural y ambiental en los habitantes de Chel, Las Flores, Xesaí y comunidades circunvecinas. Asimismo, la $\mathrm{MCH}$ es autosostenible financieramente, ya que recupera los costos de operación y mantenimiento; los salarios de los operarios y la demanda son suficientes para cubrir los costos actuales. Existe voluntad por parte de los usuarios de pagar el servicio; el cobro mensual por la energía eléctrica es diferenciado en las tarifas administrativas y de alumbrado público. En las tres comunidades fundadoras de la $\mathrm{MCH}$ la tarifa administrativa es $Q .12,00$, tarifa de alumbrado público $Q .8,00$, y costo $\mathrm{kW} / \mathrm{h}$ consumido $Q .1,00$, mientras que para las siete comunidades recientemente interconectadas la tarifa administrativa es Q. 12.50, tarifa de alumbrado público Q.12.50, y el costo por kW/h consumido Q.1.00; esto debido a que las primeras tres comunidades aportaron alrededor de 80 jornales de mano de obra no calificada y materiales locales en la construcción de la $\mathrm{MCH}$.

Los impactos sociales se han obtenido por la capacidad de gestión de los líderes de Chel, lo que ha permitido acceso a los servicios de energía eléctrica, agua potable, alcantarillado y tratamiento de aguas negras, servicios de salud, educación, estufas mejoradas, puente vehicular, salón comunal, entre otros. Los principales servicios existentes en la comunidad de Chel en el 2017 gráficamente se muestran en las Figuras $\underline{5}, \underline{6}, \underline{7}$ y $\underline{8}$. 


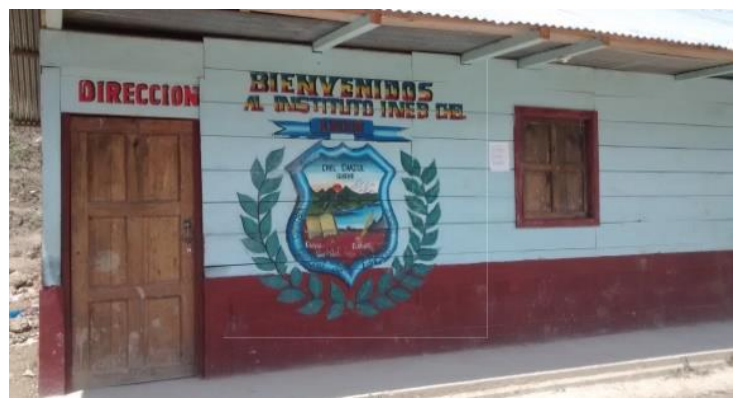

Figura 5. Educación Básica, Aldea Chel. Fuente: trabajo de campo.

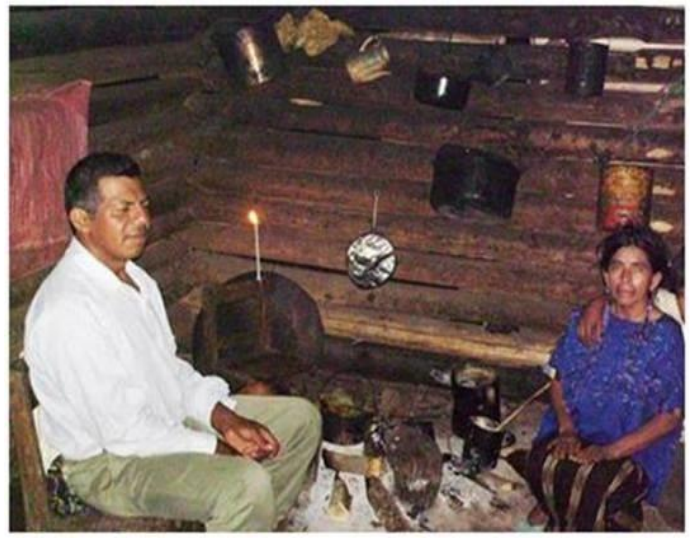

Figura 7. Situación antes de la energía eléctrica. Fuente: ASOCHEL.

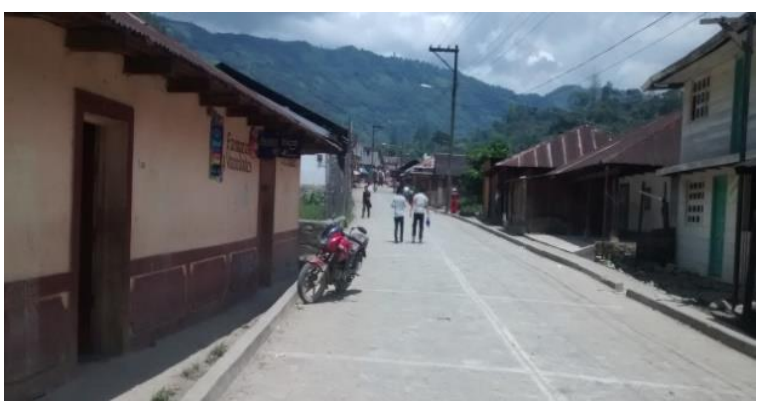

Figura 6. Calle Pavimentada. Fuente: trabajo de campo.

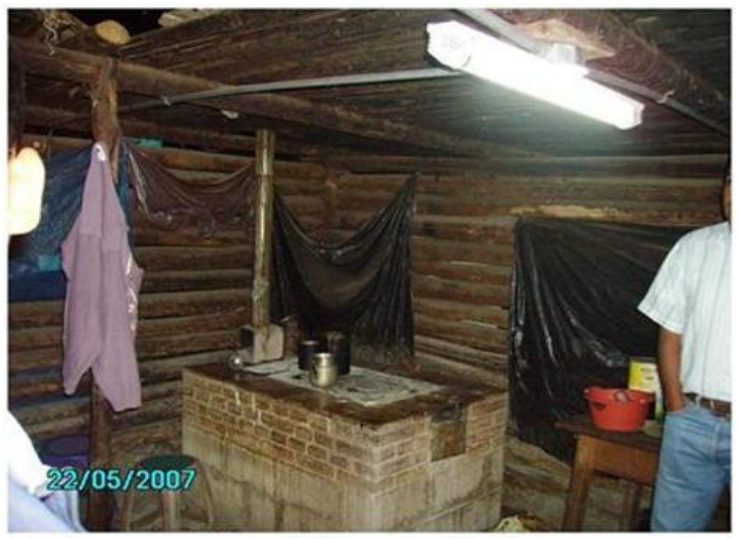

Figura. 8. Utilización de la energía eléctrica. Fuente: ASOCHEL.

En el plano ambiental, la MHC se encuentra dentro de la "Zona de Usos Múltiples" de la Reserva Biósfera Ixil Visis-Caba; técnicamente es viable, de acuerdo con la evaluación de impacto ambiental, debido a que las obras y su funcionamiento no provocan alteraciones en las condiciones ecológicas. En relación con la gestión local de los recursos naturales, se produce un servicio benéfico a partir de disponer de los recursos energéticos de Chel y reducirse el uso de derivados del petróleo y de emisiones de bióxido de carbono por la quema de leña.

En Chel existen reglas y prácticas de conservación que constituyen la institucionalidad comunitaria. Como norma general aceptada, difundida y respetada se establece no cortar árboles en parcelas de otros dueños. Cada quien es responsable de su propia parcela y pueden disponer de ella de la manera que considere más útil y provechosa, lo cual coincide con los planteamientos de Ostrom (1990) en relación con los acuerdos de los bienes comunes. Se puede percibir una ética conservacionista emergente dentro del discurso de los líderes comunitarios por evitar la tala de árboles y realizar actividades de conservación. 120

Candelario Elías Cruz Herrera, Estuardo Lara Ponce y Benito Ramírez Valverde 
La principal conducta ambiental que muestran los pobladores es de interés por conservar los recursos naturales. La generación de energía y la cobertura forestal que se mantiene, se debe a la ASOCHEL. Los habitantes se han dado cuenta de que obtienen beneficios ambientales. Esta cultura ambiental, aceptada por las nuevas generaciones, se percibe en el cambio en la vida de los ixiles; la relación entre la población y el bosque es aún más estrecha al involucrarse las mujeres en la reforestación, manejo y protección de especies.

La microcuenca de los ríos Chel y Xesayí tiene potencial para desarrollar producción forestal, agroforestal y frutal en un $100 \%$. Los líderes comunitarios y pobladores de Chel participan en la plantación de especies forestales nativas que impulsa la Hidroeléctrica Xacbal, como el "guayabillo" (Platanus mexicana), "cushín" (Inga oerstediana), "paterna" (Inga sapindoides), "chicharro" (Quercus lancifolia), "matabuey" (Lonchocarpus spp.), "cortez" (Ulmus mexicana) y "jaboncillo" (Sapindus saponaria). Además de frutales como la naranja, limón, papaya y aguacate; y de uso agroforestal, como el café y la "pacaya" (Chamaedorea spp). Estas acciones de corte agroforestal se encaminan a la protección de las microcuencas.

Otro beneficio ambiental es la generación de energía limpia y el cambio en el patrón de iluminación tradicional basado en el de consumo de leña y de combustibles fósiles (ocote, candelas y gas), que contribuye a la reducción de efectos contaminantes para la salud, que trascienden como acciones orientadas a mitigar el cambio climático (Figura $\underline{\text { 9). }}$.

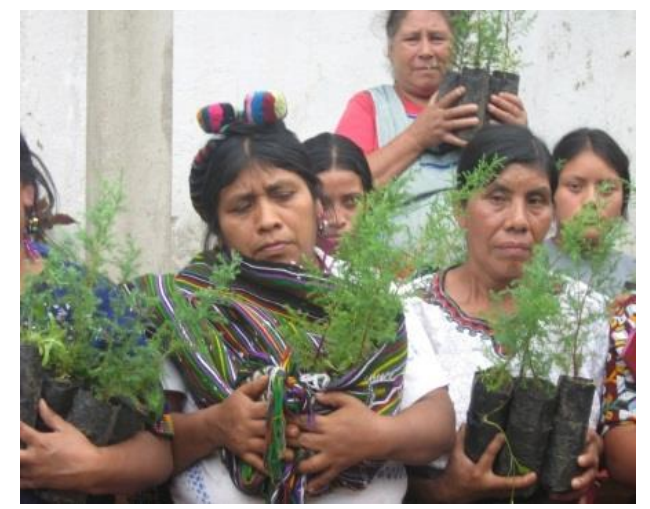

Figura 9. Mujeres en la reforestación Cuenca río Chel, 2012. Fuente: ASOCHEL.

La gestión del medioambiente utiliza y maneja los recursos naturales mediante acciones que garantizan la mejora del territorio, como la conservación y restauración de áreas y el desarrollo de tecnologías limpias. En la actualidad, la energía eléctrica es una necesidad de la que no se puede prescindir, y va en aumento, razón por la que se debe asegurar una gestión sostenible. 
El pueblo ixil, en la promoción de los valores mayas, ha trascendido con la reconstrucción social comunitaria, pese a los acontecimientos que vivieron. Se han fortalecido las comunidades vecinas fomentado la recuperación y práctica de la espiritualidad maya, revalorado el impulso de la agricultura orgánica como base de la soberanía alimentaria y de la práctica ancestral del pensamiento ixil.

La planta hidroeléctrica comunitaria es una experiencia exitosa que debe articularse a las políticas públicas, como la incorporación de la energía en la planificación municipal, siendo el Instituto de Fomento Municipal (INFOM) el ente que debiera contar con asesoría técnica en materia energética.

\section{Conclusiones}

La hidroeléctrica comunitaria es ejemplo de desarrollo para las microrregiones IV y V, en la región maya Ixil de Chajul. El proyecto inicialmente contribuyó a la reducción de la pobreza, la consolidación de la paz y la protección del medioambiente, al aprovechar el potencial de los recursos hídricos locales.

Las alianzas estratégicas de cooperación interinstitucional fueron múltiples y determinantes y varios fueron los cooperantes nacionales e internacionales para el desarrollo que aportaron recursos para materializar este esfuerzo. La gestión ambiental local significó el logro de un proceso incluyente y participativo inicial, hasta la operación de la planta hidroeléctrica. La electrificación rural cambió la vida a la comunidad, situación que se tradujo en la apropiación del proceso de desarrollo por la población ixil y la mejora de las capacidades técnicas para el manejo de la hidroeléctrica.

Las acciones emprendidas en su conjunto significaron aprendizajes útiles para otras iniciativas, como la Asociación Hidroeléctrica de Desarrollo Integral Norte del Quiché (ASHDINQUI), que en julio de 2014 inauguró su propia $\mathrm{MCH}$, que aporta 90 kilovatios (kW), beneficiando a 150 familias, también en un sistema aislado a la red nacional.

Los desafíos de la hidroeléctrica comunitaria a diez años de funcionamiento no solo consisten en consolidar el uso y consumo energético con principios de sustentabilidad. La construcción de la $\mathrm{MCH}$ es un potencial para el desarrollo local y significa un proyecto estratégico que brinda un mejor estilo de vida así como capacidad de gobernarse a sí mismos; además, puede llevar a encadenamientos productivos e influir en nuevas capacidades de gestión en las comunidades mayas.

122

Candelario Elías Cruz Herrera, Estuardo Lara Ponce y Benito Ramírez Valverde

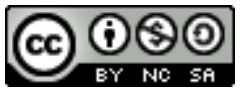

Revista Economía y Sociedad by Universidad Nacional is licensed under a CreativeCommons Reconocimiento-NoComercial- 


\section{Referencias}

Academia de Lenguas Mayas de Guatemala. (2008). Monografía Maya Ixil. (1ra ed.). Guatemala. Editorial Maya Na'oj

Akkeren, R. (2005). Introducción en la cultura maya. Ixil Lugar del Jaguar: Historia y Cosmovisión Ixil. Cooperación Alemana para el Desarollo/Serviprensa S.A: Guatemala. Recuperado de: https://es.scribd.com/doc/283458368/Ixil-Lugar-Del-Jaguar

Asociación Hidroeléctrica Chelense. (2017). Instrumentos administrativos internos. Aldea Chel, Chajul él Quiché Guatemala.

Área de Derechos Colectivos Ambientales de Pueblos Indígenas. (2006). Normas indígenas sobre el uso del agua, el bosque y la vida silvestre Maya Ixil. San Gaspar Chajul, Quiché, Guatemala.

Centro de Acción Legal - Ambiental y Social de Guatemala. (2004). Compendio de leyes y decretos. Ley que declara área protegida la Reserva de la Biosfera Ixil, Visis-Caba, ubicada en el departamento de Quiche. Guatemala.

Comisión de Esclarecimiento Histórico. (1999). Guatemala memoria del silencio. Oficina de Servicios para Proyectos de las Naciones Unidas (UNOPS), Guatemala. Recuperado de: http://www.centrodememoriahistorica.gov.co/descargas/guatemala-memoriasilencio/guatemala-memoria-del-silencio.pdf

Consejo Municipal de Desarrollo. (2010). Plan de Desarrollo Municipal (2011-2025). Chajul, el Quiché, Guatemala. Recuperado de: https://www.google.com.mx/url?sa=t\&rct=j\&q=\&esrc=s\&source=web\&cd=1\&cad=rj a\&uact=8\&ved=0ahUKEwicvcyE5bXcAhVQKawKHe xCDIQFggoMAA\&url=http\%3A\% 2F\%2Fwww.segeplan.gob.gt\%2Fnportal\%2Findex.php\%2Fbibliotecadocumental\%2Fcategory\%2F62-quiche\%3Fdownload\%3D263\%3Apdmchajul\&usg=AOvVaw0JUAi9mVQU31I 7pbBDAu2

De León, M. (2006). Las fuentes de poder del movimiento evangélico en Nebaj, el Quiché. Tesis de Licenciatura en Ciencias Políticas con Orientación en Análisis y Prospectiva. Universidad Rafael Landívar, Facultad de Ciencias Políticas y Sociales, Guatemala. 
Durocher, B. (2002). Los dos derechos de la tierra: La cuestión agraria en el país Ixil. Tomo 3. Guatemala: Facultad Latinoamericana de Ciencias Sociales, FLACSO-Guatemala. Recuperado de https://books.google.co.cr/books/about/Los dos derechos de la tierra.html?id=D ry5AAAAIAAJ\&redir esc $=y$

Fundación Solar. (2010). Sistematización de la Experiencia del Proyecto Hidroeléctrico Chel. Departamento de Quiché, Guatemala.

Gall, F. (1976). Diccionario geográfico de Guatemala (compilación critica). Tomo I. (2da ed.). Guatemala: Instituto Geográfico Nacional. Recuperado de http://biblioteca.oj.gob.gt/digitales/26558.pdf

Hamui-Sutton, A. \& Varela-Ruiz, M. (2013). La técnica de grupos focales. Investigación en educación médica, 2(5), 55-60. Recuperado de http://www.scielo.org.mx/scielo.php?script=sci_arttext\&pid=S200750572013000100009\&Ing=es\&tlng=es.

Holdrige, R. (1982). Ecología basada en zonas de vida (traducción al inglés por Jiménez, S.H.). San José, Costa Rica: Editorial IICA.

Instituto de Agricultura, Recursos Naturales y Ambiente-Universidad Rafael Landívar. (2009). Perfil Ambiental de Guatemala 2008-2009: las señales ambientales críticas y su relación con el desarrollo. Guatemala: URL- IARNA. Serie Perfil Ambiental. Recuperado de: http://www.infoiarna.org.gt/publicacion/perfil-ambiental-deguatemala-2008-2009-las-senales-ambientales-criticas-y-su-relacion-con-eldesarrollo/

Kaimowitz, D. (2001). Cuatro medio verdades: la relación bosques y agua en Centroamérica. Revista Forestal Centroamericana, (33), 6-10. Recuperado de http://bco.catie.ac.cr/portal-revistas/index.php/RRNA/article/view/759

Kawulich, B. (2005). La observación participante como método de recolección de datos. Forum: Qualitative Social Research, 6 (2) Art. 43. Recuperado de http://diverrisa.es/uploads/documentos/LA-OBSERVACION-PARTICIPANTE.pdf

124

Candelario Elías Cruz Herrera, Estuardo Lara Ponce y Benito Ramírez Valverde

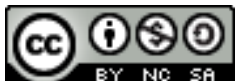

Revista Economía y Sociedad by Universidad Nacional is licensed under a CreativeCommons Reconocimiento-NoComercial- 
Ley de Protección y Mejoramiento del Medio Ambiente. (1986). Decreto 68-86 del Congreso de la República Guatemala: Ley de Protección y Mejoramiento del Medio Ambiente. Recuperado de https://www.preventionweb.net/files/27701_gtleyproteccionmedioambiente6886\% 5B1\%5D.pdf

Merino, L. (2014). Perspectivas sobre la gobernanza de los bienes y la ciudadanía en la obra de Elinor Ostrom. Revista Mexicana de Sociología, (76), 77-104. Recuperado de http://www.scielo.org.mx/pdf/rms/v76nspe/v76nspea4.pdf

Ministerio de Cultura y Deportes de Guatemala. (2001). Parque Nacional y la arquitectura vernácula del Triángulo Ixil, en Quiché, Guatemala. Guatemala: Documento de la UNESCO para Declaratoria de Patrimonio Mundial Nominación de Propiedades para la inclusión en la lista de Herencia Mundial.

Ministerio de Salud Pública y Asistencia Social. (2017). Censo Poblacional de la Aldea Chel. Guatemala: Chajul él Quiché.

Molina, R. (2012). Hidroenergía: Conflicto y solución a una necesidad de país. Recuperado de: http://digi.usac.edu.gt/bvirtual/informes/informes2012/INF-2012-08.pdf

Muriel, R. (2005). Orígenes de la problemática ambiental. Revista Ide@s Sostenible: Espacio de reflexión y comunicación en desarrollo sostenible. Año 2, No. 9, 1-6. Recuperado de: https://upcommons.upc.edu/bitstream/handle/2099/248/012 ORIGEN PROBLEM AMBIENTAL Muriel CAST.pdf

Oficina de Derechos Humanos del Arzobispado de Guatemala. (2000). Memoria, Verdad y Esperanza (Versión popular del informe REMHI: Guatemala: Nunca Más). Arzobispado de Guatemala. Oficina de Derechos Humanos, Guatemala. Recuperado de: http://www.odhag.org.gt/pdf/tomo 1.pdf

Organización Latinoamericana de Energía (OLADE). (2012). Estudio: Diálogo sobre el tratamiento de aspectos Socioambientales en Proyectos Hidroeléctricos en Latinoamérica. Quito Ecuador.

Ostrom, E. (1990). Reflections On The Commons. In Governing the Commons: The Evolution of Institutions for Collective Action (Political Economy of Institutions and Decisions, pp. 1-28). Cambridge: Cambridge University Press. doi: https://10.1017/CBO9780511807763.003 
Política energética de Guatemala. (2013-2027). Energía para el desarrollo. Guatemala. Recuperado de http://www.mem.gob.gt/wp-content/uploads/2013/02/PE20132027.pdf

Rey, M., Conde, C. Godoy, J. Secaira, E., y Schuster, J. (1996). Estudio Técnico Reserva de la Biosfera Ixil Visís Cabá. Guatemala: Fundación Madre Selva.

126 